\title{
Expression of Cytoplasmic TFF2 Is a Marker of Tumor Metastasis and Negative Prognostic Factor in Gastric Cancer
}

\author{
Dipok Kumar Dhar, Timothy C. Wang, Riruke Maruyama, Jun Udagawa, \\ Hirofumi Kubota, Toshiyuki Fuji, Mitsuo Tachibana, Takashi Ono, Hiroki Otani, and \\ Naofumi Nagasue
}

Department of Digestive and General Surgery (DKD, HK, TF, MT, TO, NN), Department of Central Pathological Laboratory (RM), and Department of Anatomy (JU, HO), Shimane Medical University, Izumo, Japan; and Gastroenterology Division and Department of Medicine (TCW), University of Massachusetts Medical School, Worcester, Massachusetts

SUMMARY: Trefoil factor family 2 (TFF2) is a small peptide constitutively expressed in the gastric mucosa, where it plays a protective role in restitution of gastric mucosa. TFF2 has also been shown to be expressed in some gastric cancers, but its role in tumor metastasis and patient prognosis has not been examined. In this study, we examined TFF2 expression at both the mRNA and protein levels and correlated these results with the clinicopathologic characteristics and prognosis of gastric cancer patients. Among the 144 curatively resected samples, $43(30 \%)$ were positive for TFF2. TFF2 expression was preferentially observed in the infiltrating tumor cells sparing the superficial cells. Significantly increased expression of TFF2 was noted in large tumors of the diffuse type. An increased prevalence of TFF2 expression was also found in tumors with advanced T and N stage and in patients with lymphatic and venous invasion. Accordingly, patients with TFF2-expressing tumors had a significantly worse disease-free survival, and in multivariate analysis, this finding remained significant as an independent prognostic factor. Taken together, our results suggest that TFF2 expression may play a role in gastric cancer invasion and as such could be a useful target for therapeutic intervention. (Lab Invest 2003, 83:1343-1352).

$$
T^{\text {hat }}
$$
he trefoil factor family (TFF) peptides are a group of protease-resistant proteins that all contain one or more trefoil domains, a characteristic threeleafed structure that has given the peptide family its name (Wright et al, 1997). There are three known trefoil factors: TFF1 (also known as pS2) is an estrogenresponsive peptide but expresses abundantly in the surface epithelium of the stomach; TFF2 (also known as spasmolytic polypeptide [SP]) is expressed in mucous neck cells of the gastric mucosa and Brunner's glands of duodenum; and TFF3 (also known as intestinal trefoil factor or ITF) is expressed in the mucussecreting goblet cells of the intestine. The genes encoding these TFFs all are clustered within a tandem of $55 \mathrm{~kb}$ on human chromosome 21q22.3 and are thought to have overlapping biologic activities (Seib et al, 1997). Trefoil peptides are involved in several biologic functions, including gastrointestinal cytoprotection, cellular proliferation, and increase cell motility (Hoosein et al, 1989; Jørgensen et al, 1982; Playford et al, 1995). The role of TFFs in tumorigenesis still

\section{DOI: 10.1097/01.LAB.0000085190.99749.15}

Received June 4, 2003.

Address reprint requests to: Dr. Dipok Kumar Dhar, Department of Digestive and General Surgery, Shimane Medical University, Izumo 693-8501, Japan. E-mail: nigeka33@shimane-med.ac.jp remains controversial; in general, TFF1 seems to function as a gastric-specific tumor suppressor gene, whereas TFF3 has been postulated to enhance tumor progression by increasing the cellular invasion and metastasis (Lefebvre et al, 1996; Yamachika et al, 2002). Although it was reported that TFF2 expression is associated with the development of gastric cancer formation in mouse, its precise role in gastric carcinogenesis remains obscure (Wang et al, 1998, 2000).

The majority of the studies on the TFFs have focused on their role in preventing cryoprobe- or indomethacin-induced gastric mucosal injury. Among the three TFFs, TFF2 deserves special attention because of its rapid induction (within 30 minutes) in the vicinity of injured mucosa and its clear role in the restitution of breached mucosa by increasing cellular motility (Alison et al, 1997). Early reports suggested that TFF2 works as a mitogen as it increased thymidine incorporation in MCF-7 and HCT-116 cells (Hoosein et al, 1989). However, later studies pointed to a primary role as a motogen. In an in vitro wounding assay, TFF2 stimulated cell migration in a dosedependent manner and also induced vertical invasion of HT29 cells into type I collagen gel motility/invasion assay (Playford et al, 1995). More recently, studies in TFF2-deficient mice generated through targeted gene disruption showed evidence of decreased gastric cell 
proliferation and increased susceptibility to gastric mucosal injury induced by indomethacin treatment (Farrel et al, 2002). The normal expression of TFF2 in the proliferative zone of gastric mucosa, along with its demonstrated roles as a motogen and in suppressing acid secretion, raised the possibility that TFF2 might have a role in neoplastic transformation or tumor progression.

To date, only a few studies have evaluated expression of TFF2 in a limited number of gastric carcinomas (Halldorsdottir et al, 2003, Kirikoshi and Katoh, 2002; Leung et al, 2002; Machado et al, 2002; Theisinger et al, 1991). Expression of TFF2/SP in stomach cancer was first reported by Theisinger et al (1991). In addition, more recent reports have indicated that increased TFF2 expression can be detected in patients with Helicobacter-associated chronic fundic gastritis and is often present in association with dysplasia. TFF2-expressing cells are also up-regulated in the stomachs of Helicobacter-infected mice and seem to give rise to invasive cancerous lesions (Wang et al, 1998, 2000). However, the precise correlation of TFF2 expression with metastasis related clinicopathologic features in human gastric cancer patients and the overall impact on patient prognosis have not been evaluated. To address these questions, we evaluated the expression of TFF2 at both the MRNA and protein levels in a large series of curatively resected gastric carcinoma patients. Our findings indicate that TFF2 expression is frequently up-regulated in diffuse gastric cancers and correlates with decreased survival.

\section{Results}

\section{TFF2 Expression in Benign and Malignant Tissues}

Evaluation of TFF2 immunohistochemical staining was done blindly and independently by two of the authors (DKD and FT), and any discordant result was settled by using a conference microscope. TFF2 immunostaining was observed in all of the investigated normal mucosa samples, and in these tissues, staining largely was restricted to the neck cells of the gastric glands, sparing the superficial foveolar epithelial cells and deep glands (Fig. 1a). In contrast to this normal pattern, in cancer patients, the lower half of the fundic
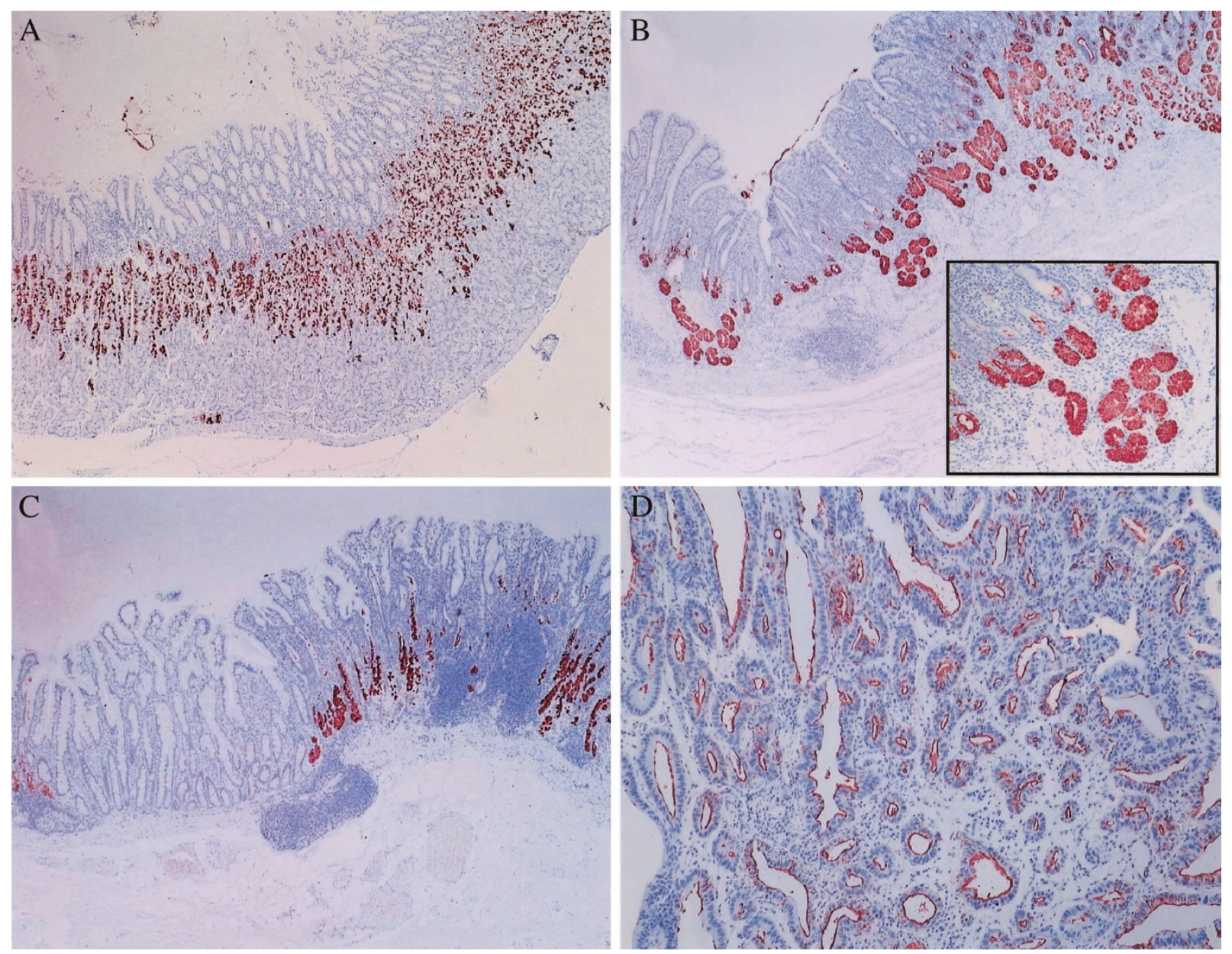

Figure 1.

Normal pattern of trefoil factor family 2 (TFF2) expression in gastric mucosa (a), spasmolytic polypeptide (TFF2)-expressing metaplasia (SPEM; b), intestinal metaplasia (c), and intestinal-type cancer (d) are shown. Normal staining for TFF2 was observed in the isthmus region of the gastric glands, whereas the SPEM lineage of cells was located in the lower half of the fundic glands. The SPEM cells had abundant mucous granules and had morphologic similarity with the Brunner's gland of the duodenum. Regions of intestinal metaplasia were always negative, and a weak membranous staining was observed in intestinal-type gastric adenocarcinoma. 
glands were frequently positive for TFF2 in the noncancerous gastric mucosa adjacent to the tumor (Fig. 1b). These TFF2-positive fundic glandular cells had abundant mucous granules and had morphologic similarity with both antral glands and Brunner's gland of the duodenum(also know as SP-expressing metaplasia [SPEM]). Among the 144 tumors, the SPEM lineage cells were present in 135 (94\%) gastric mucosa adjacent to the tumor. In contrast, only $70 \%$ (43 of 144) of patients had intestinal metaplasia in the adjacent gastric mucosa ( $p=0.0000$, vs SPEM, F test). Frequently the SPEM lineage cells and intestinal metaplasia coexisted. Mononuclear cell infiltration was frequently noticed around the SPEM lineage cells (Fig. 1, $\mathrm{b}$ and $\mathrm{c}$ ). Mononuclear cells and stromal cells were always negative both in normal mucosa and in tumor. Occasionally, endothelial cells lining tumor-associated blood vessels were positive for TFF2. Intestinal metaplastic mucosa adjacent to the tumor tissue was negative for TFF2 (Fig. 1c).

In terms of cellular localization, TFF2 staining was observed as cytoplasmic, apical, or membranous staining and mixed type of staining. In malignant tissues, strong and distinct staining for TFF2 was seen in the cytoplasm predominantly in the diffuse type of cancers, whereas weak membranous staining was the characteristic feature of the intestinal tumors (Fig. 1d). In most of the tumors positive for TFF2, strong cytoplasmic staining was preferentially observed in the invasive cells sparing the superficial tumor cells (Fig. 2, a and b). TFF2 expression was particularly notable in cases in which the tumor cells had invaded beyond the muscularis mucosa layer (Fig. 2c). Moreover, the metastatic tumor cells in the regional lymph nodes of the TFF2-positive cases were strongly stained for TFF2 (Fig. 2d). The staining was confined to the metastatic tumor cells and was somewhat heterogeneous, with a large number of positive cells in the periphery of the tumor nodule.

TFF2 mRNA expression was detected as a single band by the Northern blot analysis (Fig. 3). There was a significant correlation between the results obtained by the Northern blot and immunohistochemical analyses ( $p=0.0007$, Spearman's rank correlation test).
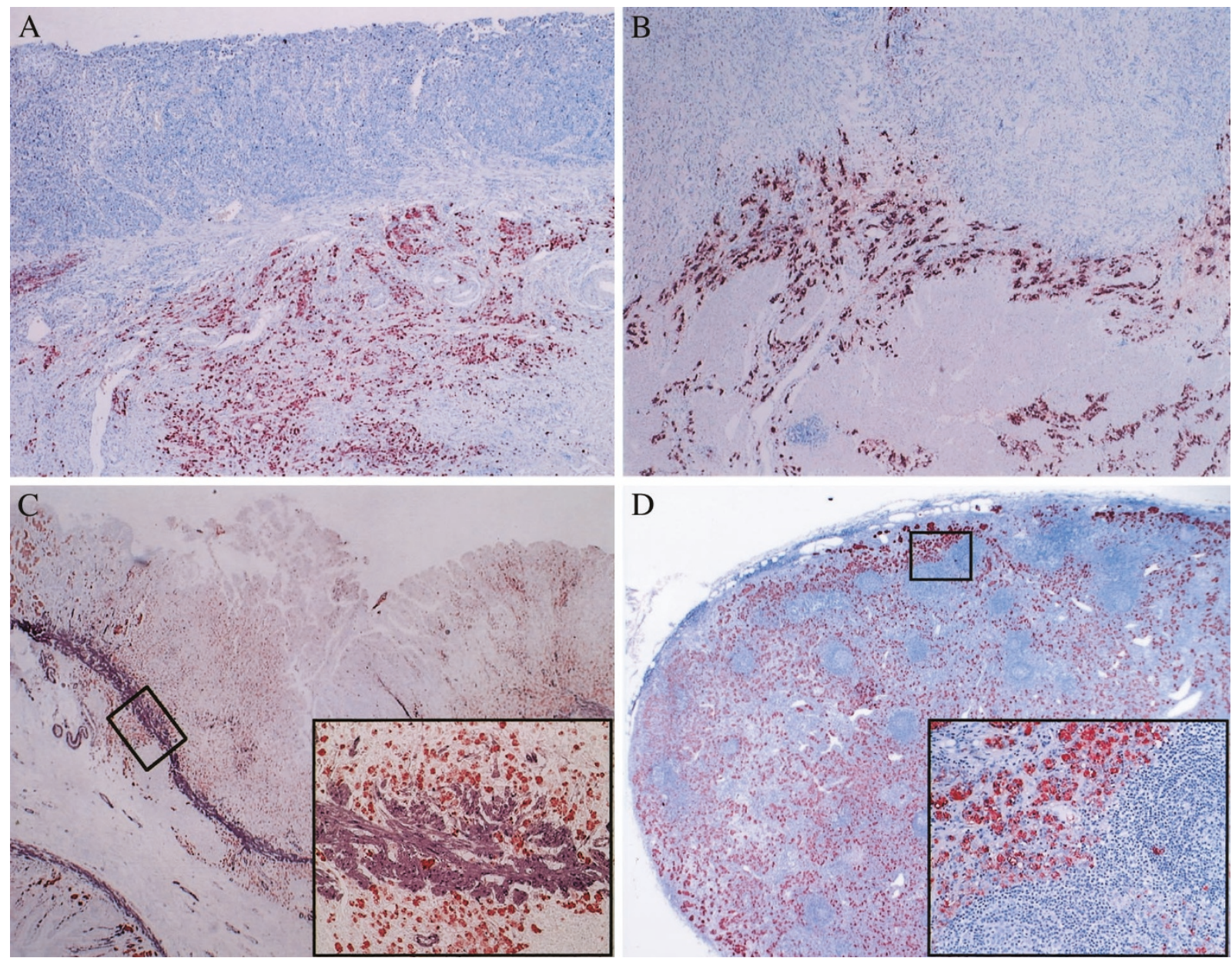

Figure 2.

Representative sections of diffuse-type tumors and metastatic lymph node (LN) with strong cytoplasmic staining in the infiltrative tumor cells are shown. a and $b$, Although sparse superficial tumor cells had staining for TFF2, in most of the instances, strong homogeneous staining was noticed only in the infiltrating tumor cells of both small and large tumors. c, Double immunostaining with $\alpha$-SMA (purple) and TFF2 (red) shows that TFF2-positive tumor cells are invading the muscularis mucosa layer (no counterstaining). $d$, A regional metastatic LN from a patient with TFF2-positive tumor was also positive for TFF2. Original magnification, $\times 40$ in $\mathrm{a}$ and $\mathrm{b}, \times 20$ in $\mathrm{c}, \times 200$ in $\mathrm{c}$ and $\mathrm{d}$ inset, $\times 40$ in $\mathrm{d}$. 


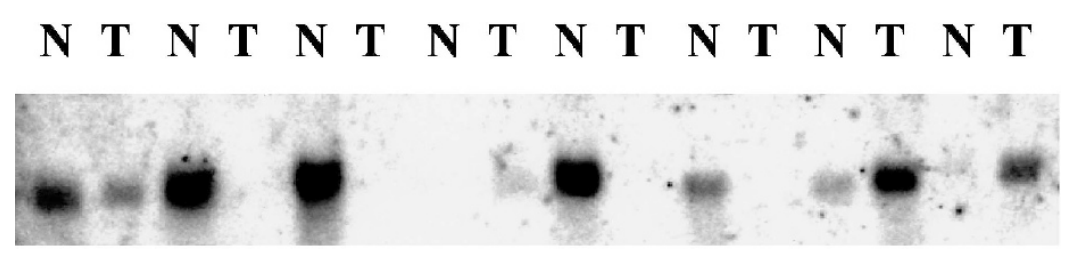

Figure 3.

RNA blot showing TFF2 mRNA expression in normal mucosa adjacent to the tumor and tumor samples. Only a few tumors had TFF2 expression, whereas most of the normal samples were positive for TFF2.

Among the 31 studied samples, 20 were negative by the staining, and of these 20 cases, 19 were found negative by the Northern blot analysis, whereas among the 11 positive cases by the staining, 7 were positive by the Northern blot and 4 were negative. In selected cases, in situ hybridization was done in serial tissue sections to compare between the mRNA and protein expressions. Although in most of the examined tumors, mRNA and protein were detected in the same areas, this was not always true. In general, mRNA was expressed more diffusely than the protein expression. In the normal mucosa, mRNA expression was not restricted in the neck cells but extended superficially to some extent in the foveolar cells (Fig. 4, a and b). In the tumors, both mRNA and TFF2 protein were localized in the invasive cells (Fig. 4, $c$ and d), but sometimes TFF2 mRNA expression was noticed in more superficial cells that had no protein expression. Occasionally, instead of strong TFF2 protein expression in the tumor cells, mRNA could not be detected in these cells.

\section{Correlation with Clinicopathologic Parameters}

Tumors having cytoplasmic or mixed type of staining were considered to be positive for TFF2, and among 144 cases, $43(29.8 \%)$ cases were positive for TFF2. There was no correlation between TFF2 positivity and the sex or age of the patient. There was a strong correlation $(p<0.001)$ with the differentiation status of the tumor, with an increased prevalence of expression in the diffuse type of tumors. Fifty-two percent (52\%) of the diffuse tumors were positive, whereas only $15 \%$ of the intestinal tumors were positive for TFF2. With respect to early gastric cancers, only two cases were positive, and both of them were of diffuse tumors (2 of 7). Expression of TFF2 increased with the advancement of the $T(p=0.0011)$ and $N$ stage $(p=0.0001)$ of the tumor. Also, a similar correlation was observed with the lymphatic $(p=0.0007)$ and venous vessel invasions $(p=0.0189)$. The average tumor size of the TFF2-positive tumors $(6.7 \pm 3.6 \mathrm{~cm}$, mean $\pm \mathrm{sd})$ was
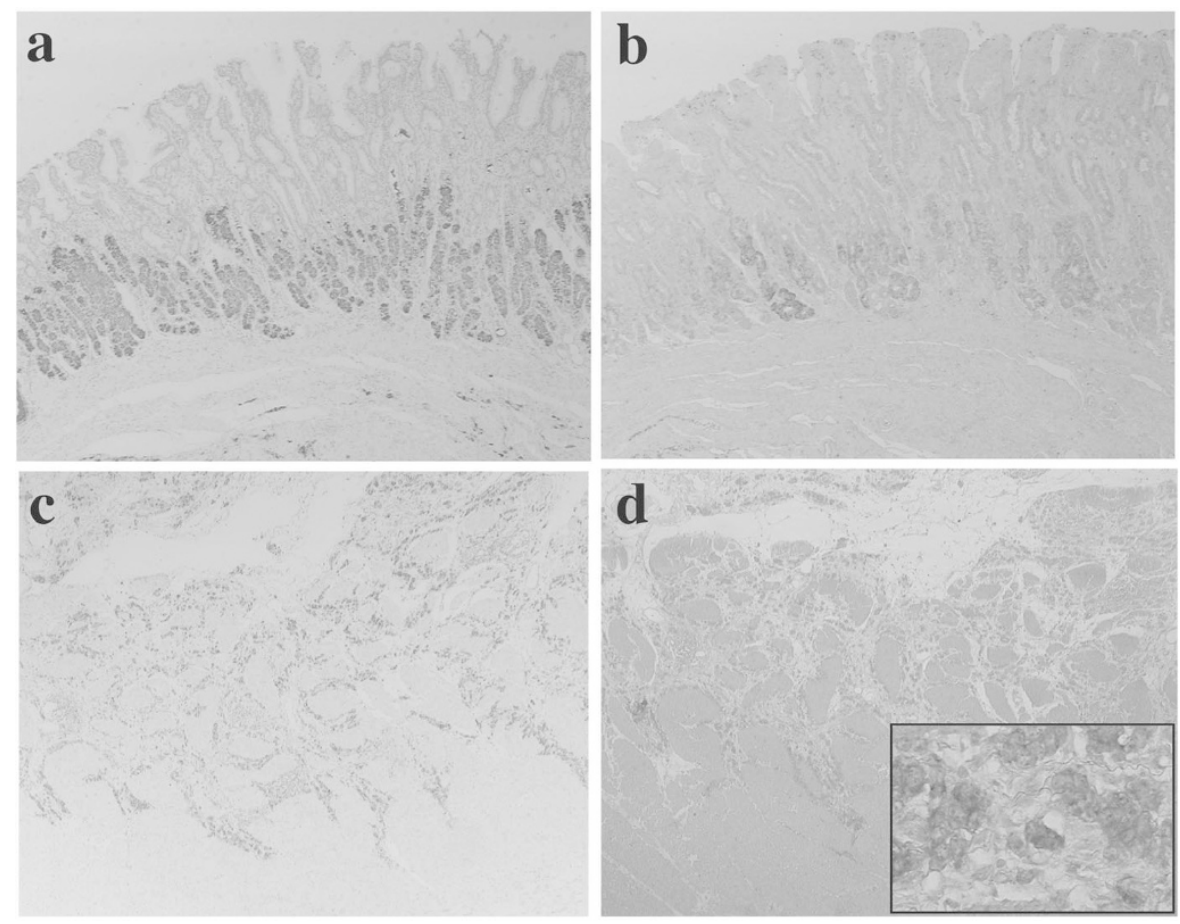

Figure 4.

Serial sections showing TFF2 mRNA (purple, with no counterstaining) and protein (red) expressions in gastric mucosa adjacent to the tumor and tumor region inset $\times 200$ ) are shown. Both mRNA and TFF2 protein were localized in the same region of the gastric mucosa and infiltrating tumor cells. Original magnification, $\times 40$, $\times 200$ in inset. 
significantly ( $p=0.0068)$ higher than those of negative ones $(4.5 \pm 2.5 \mathrm{~cm}$, mean $\pm \mathrm{sd}$; Table 1$)$.

\section{Relationship Between TFF2 Status and Patient Survival}

The median follow-up period was 47.2 months. TFF2 expression had a significant ( $p<0.0001$, log rank test) impact on patient prognosis only in terms of the disease-free survival (Fig. 5) and had a trend ( $p=$ 0.0973 , log rank test) of poor overall survival in the TFF2-positive cases but did not reach statistical significance. The 5-year overall survival rates of patients with TFF2-negative and -positive status were $66.8 \%$ and $54.8 \%$, whereas the 5-year disease-free survival rates for TFF2-negative and -positive patients were $86.1 \%$ and $53.4 \%$, respectively. A multivariate analysis was done to single out the independent prognostic factors, and all of the conventional prognostic variables along with the TFF2 were included in the analysis. Among the several factors analyzed, only the tumor stage, TFF2 expression, and amount of intraoperative blood loss remained significant for the disease-free survival (Table 2).

\section{Discussion}

Gastric carcinoma remains a generally lethal disease with a $<25 \%$ 5-year survival rate, yet early gastric cancer (neoplastic lesions that have not yet penetrated through the muscularis layer of the stomach) is often curable and carries with it a much better prognosis. It remains unclear whether advanced gastric cancers, which by definition have invaded through the muscularis, are biologically distinct from early gastric cancer or simply a later stage of a similar lesion. In any case, the invasive phenotype is required for progression to advanced gastric cancer and a prerequisite for the development of metastasis. Few genes have been identified to serve as useful prognostic markers for invasive gastric cancers. Our study suggests that TFF2 (formerly known as spasmolytic polypeptide) may represent such a prognostic marker. Our work suggests that the TFF2 gene is expressed preferentially in invasive gastric cancers and is strongly associated with tumors of diffuse histology. Immunoreactivity for TFF2 was also found in a retrospective analysis to be associated with a worse prognosis.

Table 1. Correlation of TFF2 with the Clinicopathologic Factors

\begin{tabular}{|c|c|c|c|}
\hline & TFF-2 $(+$ ve $)(n=43)$ & TFF-2 $(-$ ve $)(n=101)$ & $p$ Value \\
\hline Age (years) & $63.7 \pm 11.5$ & $66.7 \pm 12.0$ & 0.721 \\
\hline \multicolumn{4}{|l|}{ Sex } \\
\hline Male & 30 & 66 & \multirow[t]{2}{*}{0.747} \\
\hline Female & 13 & 35 & \\
\hline Tumor size $(\mathrm{cm}$, mean $\pm \mathrm{SD})$ & $6.7 \pm 3.6$ & $4.8 \pm 2.5$ & $0.0068^{a}$ \\
\hline \multicolumn{4}{|l|}{ Location } \\
\hline Upper & 9 & 31 & \multirow[t]{3}{*}{0.0929} \\
\hline Middle & 20 & 28 & \\
\hline Lower & 14 & 42 & \\
\hline \multicolumn{4}{|l|}{ Differentiation } \\
\hline Well & 13 & 69 & \multirow[t]{2}{*}{$<0.001^{b}$} \\
\hline Poor & 30 & 32 & \\
\hline \multicolumn{4}{|l|}{ N stage } \\
\hline 0 & 10 & 56 & \multirow[t]{4}{*}{$0.0001^{b}$} \\
\hline 1 & 20 & 37 & \\
\hline 2 & 7 & 7 & \\
\hline 3 & 6 & 1 & \\
\hline \multicolumn{4}{|l|}{ T stage } \\
\hline 1 & 2 & 31 & \multirow[t]{4}{*}{$0.0011^{b}$} \\
\hline 2 & 18 & 38 & \\
\hline 3 & 20 & 30 & \\
\hline 4 & 3 & 2 & \\
\hline \multicolumn{4}{|l|}{ Lymphatic invasion } \\
\hline 0 & 1 & 26 & \multirow[t]{4}{*}{$0.0007^{b}$} \\
\hline 1 & 9 & 26 & \\
\hline 2 & 14 & 24 & \\
\hline 3 & 19 & 25 & \\
\hline \multicolumn{4}{|l|}{ Venous invasion } \\
\hline 0 & 2 & 30 & \multirow[t]{4}{*}{$0.0189^{b}$} \\
\hline 1 & 10 & 19 & \\
\hline 2 & 16 & 38 & \\
\hline 3 & 15 & 23 & \\
\hline
\end{tabular}

${ }^{a}$ Mann-Whitney $U$ test.

${ }^{b}$ Fisher's exact test. 


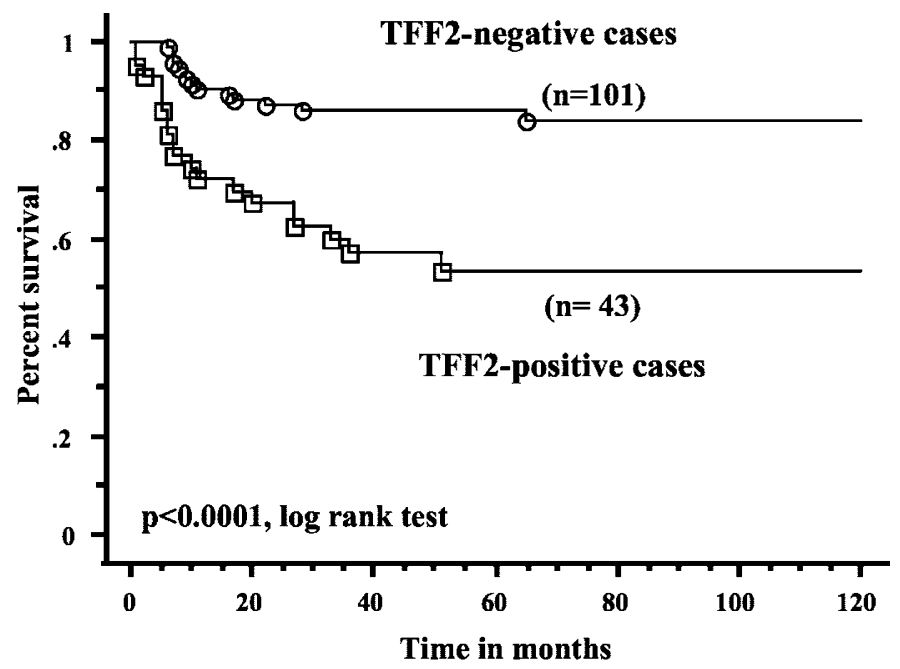

Figure 5.

Disease-free survival curves stratified by the TFF2 expression are shown. A significantly $(p<0.0001, \log$ rank test) worse survival was noticed in patients with TFF2-positive tumors.

Table 2. Multivariate Analysis of Prognostic Factors

\begin{tabular}{|c|c|c|c|c|}
\hline \multirow[b]{2}{*}{ Prognostic Factors } & \multicolumn{2}{|c|}{ Overall Survival } & \multicolumn{2}{|c|}{ Disease-Free Survival } \\
\hline & $\chi$ Value & $p$ Value & $\chi$ Value & $p$ Value \\
\hline UICC stage & 17.22 & $<0.001$ & 26.17 & $<0.0001$ \\
\hline TFF2 expression & 0.68 & 0.4095 & 5.34 & 0.0208 \\
\hline Intraoperative blood loss & 4.38 & 0.0363 & 4.89 & 0.0270 \\
\hline Differentiation & 0.26 & 0.6102 & 1.69 & 0.1939 \\
\hline Tumor size & 0.22 & 0.6388 & 0.01 & 0.9201 \\
\hline Venous invasion & 3.36 & 0.5001 & 0.47 & 0.4930 \\
\hline Lymphatic invasion & 5.04 & 0.1690 & 1.36 & 0.9825 \\
\hline Age & 4.89 & 0.0270 & 0.65 & 0.4197 \\
\hline Sex & 0.34 & 0.5592 & 0.00 & 0.9446 \\
\hline
\end{tabular}

To date, only a few studies have examined the expression pattern of TFF2 in gastric cancer (Halldorsdottir et al, 2003; Kirikoshi and Katoh, 2002; Leung et al, 2002; Machado et al, 2002; Theisinger et al, 1991). Furthermore, a possible role for TFF2 in progression of gastric carcinoma has never been addressed. To the best of our knowledge, this is the first study to report a correlation between TFF2 expression and the clinicopathologic features and/or prognosis of gastric cancer. In a cohort of 29 patients with early gastric cancer, Halldorsdottir et al (2003) reported that 18 (62\%) cancers were positive for TFF2, whereas Machado et al (2002) reported that TFF2 was expressed by only $10.4 \%$ of gastric cancer specimens, with a trend (but no statistical difference) of higher expression in diffuse versus intestinal type tumors. In another study, Leung et al (2002) detected TFF2 mRNA in 44\% gastric cancers; however, the clinical significance of TFF2 expression was not sought in their samples. Theisinger et al (1991) and Kirikoshi and Katoh (2002) reported that both TFF1/pS2 and TFF2/SP were strongly expressed in diffuse-type gastric cancers and in diffuse-type gastric cancer cell lines, respectively. Similarly, in our study, TFF2 expression was signifi- cantly higher in diffuse-type cancers than in intestinal type. Of note, the cellular localization for TFF2 was specific for each histologic type of cancer. In diffuse gastric cancer, TFF2 was primarily cytoplasmic in location (similar to the pattern in normal gastric glands), whereas in intestinal-type gastric cancer, there was a very weak membranous expression pattern. In contrast to the report by Halldorsdottir et al (2003), in this study, only two patients with early gastric cancer ( 2 of $33,6 \%$ ) were positive for TFF2. This discordant result could be due to the presence of a large number of intestinal-type cancers in our series (26 of 33), which when stained for TFF2 were mostly either luminal or membranous staining. In our study, tumors having luminal or membranous staining were considered to be negative for TFF2 because most of the positive invasive cancer cells and SPEM lineage cells had cytoplasmic staining. The significance of the cellular localization of TFF2 will require further investigation. If only the cytoplasmic TFF2 expression is considered when compared with the adjacent gastric mucosa, then it seems that TFF2 expression is downregulated in a large number of samples. The exact reason for this change in the expression pattern of 
TFF2 in carcinomas is yet to be evaluated. Park et al (2000) reported that loss of heterozygosity is responsible for down-regulation of TFF1 gene in gastric carcinomas, and also a similar mechanism may be responsible for down-regulation of TFF2 in gastric carcinomas and needs further studies to reveal the exact mechanism.

TFF peptides are protease-resistant peptides that are believed to play a role largely in gastric cytoprotection. However, on the basis of the knockout models, it is likely that there are additional functions specific to each of the TFF members. Thus, whereas all of the trefoils seem to be up-regulated in response to injury to the gastrointestinal mucosa, TFF2 is unique in that it is induced the most rapidly (within 30 minutes). At least one study has indicated that induction of TFF2 expression precedes that of gastrointestinal growth factors such as epidermal growth factor and transforming growth factor- $\alpha$ (Alison et al, 1997). In addition, TFF2 is expressed by mucous neck cells in close approximation to the proliferative zone in both the gastric antrum and body. Several studies have suggested that TFF2 may promote cellular proliferation (Hoosein et al, 1989; Otto et al, 1996); indeed, TFF2-deficient mice have been reported to decrease gastric mucosal proliferation (Farrel et al, 2002).

Taken together, the increase in TFF2 expression noted in the larger, more advanced tumors raises the intriguing possibility that TFF2 might play some role in cellular proliferation and/or the growth of gastric cancers. Consistent with this model, we found that the prevalence of TFF2 expression correlated with a more advanced $\mathrm{N}$ stage and with the presence of lymphatic or vascular invasion. However, it is possible that TFF2 might contribute to tumor progression in other ways, such as cell migration, angiogenesis, or immune suppression. Indeed, several studies have supported a role with respect to cell migration and possibly invasion (Efstathiou et al, 1999; Emami et al, 2001; Graness et al, 2002).

A strength of this study lies with the specific localization of both mRNA and protein of TFF2 in the cancer cells of the invasive front of the tumor sparing the superficial luminal cancer cells. Although there was some discrepancy between the mRNA and protein expression, both mRNA and protein of TFF2 were detected in the invasive tumor cells. Also, the tumor cells inside the metastatic lymph nodes of the TFF2positive patients were strongly positive for TFF2, indicating that TFF2-positive cell lineage metastasized to the regional lymph nodes and the metastatic phenotype could be attributable to the TFF2 expression by the tumor cells. This finding further strengthens the conclusions of previous in vitro studies stating that TFF2 is intricately related with cancer cell motility and invasion (Efstathiou et al, 1999; Emami et al, 2001; Graness et al, 2002; Playford et al, 1995). In an in vitro wound-induced cell migration assay using confluent rat intestinal epithelial cells, Dignass et al (1994) showed that application of TFF2 enhanced migration of cells into the wound approximately 4-fold. In addition, Playford et al (1995) showed that TFF2 induced vertical invasion of HT29 cells into type I collagen gel in a dose-dependent manner.

Thus, several of the downstream molecular pathways connecting the trefoil peptides with the invasive phenotype of cells are becoming clear. The results of several recent studies seem to support the idea that epidermal growth factor receptors, mitogen-activated protein kinase, and adenomatous polyposis coli (APC)/E-cadherin/ $\beta$-catenins are regulated by the trefoil peptides (Efstathiou et al, 1999; Liu et al, 1997; Taupin et al, 1999). It was reported that E-cadherin expression was reduced in the restitutive monolayer epithelium of ulcers in which trefoil peptides were expressed (Hanby et al, 1996). More recently, Emami et al (2001) showed that activation of several other signaling pathways involving the cellular transformation and survival, namely, src/RhoA, PI3-K/Akt, and phospholipase $\mathrm{C} /$ protein kinase $\mathrm{C}$, play a crucial role in cellular invasion by TFFs. However, the precise mechanism by which TFF2 is able to exert these effects is currently not known. Although specific receptors have been postulated and some candidate binding proteins have been identified, further investigation is needed to clarify these pathways.

Accordingly, TFF2 expression status seems to be a good candidate to become an independent prognostic marker for disease recurrence along with other conventional prognostic factors in gastric cancer patients. Yamachika et al (2002) made a similar suggestion that the expression of TFF3 could become an important prognostic marker for gastric carcinoma patients. The results of our study indicate that TFF2 may be responsible for decreased cell-cell/substratum adhesion, cellular invasion, and poor prognosis in gastric cancer and, therefore, could be a useful target for intervention of progression of gastric cancer. Future prospective studies will be needed to define the utility of TFF2 expression with clinical behavior and outcome and its biologic role in cancer progression.

The absence of TFF2 expression in many of the early gastric cancers and intestinal metaplasias might suggest that TFF2 expression may play less of a role in the early stages of gastric carcinogenesis. However, although the numbers were small, it should be noted that an equal percentage of diffuse-type early gastric cancers (2 of 7) were positive for TFF2 immunostaining. A recent study by Schmidt et al (1999) described the presence of a TFF2-positive cell lineage with morphologic features similar to pyloric metaplasia or Brunner's glands in the duodenum that occurred in association with gastric cancer. We also found in noncancerous portions of the gastric body of our patients an altered pattern of TFF2 immunostaining that was consistent with this lesion (labeled SPEM). As reported by other authors (Halldorsdottir et al, 2003; Schmidt et al, 1999), we also found that the incidence of SPEM lineage of metaplasia was significantly higher than the incidence of intestinal metaplasia in adjacent gastric mucosa to the cancers. This may indicate a strong association between the SPEM lineage cells and gastric adenocarcinoma. Further studies will need to address the significance of this TFF2 expressing 
cell lineage and whether it represents the precursor lineage for gastric cancer.

\section{Materials and Methods}

\section{Patients and Samples}

Between February 1990 and December 1998, 111 patients who had advanced gastric cancer and were undergoing a curative resection were included in this study. Another 33 patients who were randomly selected from a pool of early gastric cancer patients were also included. Of these patients, 48 were women and 96 were men; their ages ranged from 29 to 94 years. Immediately after resection, all specimens were opened in the operating room and photographed for documentation. According to a prospective protocol, tumor and all lymph nodes (LNs) were cut at several levels and embedded in paraffin, and sections were taken for routine hematoxylin-eosin staining. An experienced pathologist checked all of these slides and documented the pathologic characteristics of the tumor and LNs. Tumor stage was defined according to the International Union Against Cancer (UICC)-Tumor Node Metastasis (TNM) (1997) staging system. Histologic classification was done according to the Lauren classification. The follow-up was complete in all patients. The data obtained at regular follow-up visits at the outpatient department were stored in a database specially designed for gastric carcinoma patients. An update inquiry about the present status of all surviving patients was made by telephone call in March 2002. In case of any recurrence, the exact date of disease recurrence was collected from the referring physician or from the physician who attended the patient for the first time for the diagnosis of the disease recurrence. All patients provided consent for use of tumor tissue for clinical research, and the University Ethical Committee approved the research protocol.

\section{Immunohistochemistry}

We used a highly specific mAb against the TFF2, which was proved effective in detecting TFF2 on paraffin sections (Elia et al, 1994; Playford et al, 1995). Representative paraffin sections containing both the normal mucosa and the invasive front of the tumor tissue were selected for the immunohistochemical staining. Also, regional metastatic LNs from selected patients were used for the immunohistochemistry. Briefly, slides were deparaffinized, rehydrated in graded alcohols, and placed in PBS solution. Antigen retrieval was done by autoclaving the slides for 10 minutes in citrate buffer $(\mathrm{pH}$ 6.0; Dako ChemMate; Dako, Glostrup, Denmark). Endogenous peroxidase activity was quenched by dipping in $3 \% \mathrm{H}_{2} \mathrm{O}_{2}$ for 30 minutes, and nonspecific binding was blocked by treating slides with normal rabbit serum for 30 minutes. Mouse anti-human TFF2 mAb (GE16C clone; Novocastra Laboratories Ltd., Newcastle, United Kingdom; 1:50 dilution) was used at $4^{\circ} \mathrm{C}$ for overnight incubation. The streptavidin-biotin peroxidase complex method was used for the immunohistochemical steps and were done with a commercially available kit (Histofine SAB-PO kit; Nichirei, Tokyo, Japan) as we described previously (Nagasue et al, 2000). Color development was done with the peroxidase substrate 3-amino-9-ethylcarbazole. Adjacent normal mucosa served as an internal positive control, and in negative control slide, the primary antibody was replaced by nonimmune serum.

Double immunohistochemical staining was performed to simultaneously localize $\alpha$-smooth muscle actin ( $\alpha$-SMA) and TFF2 using a labeled-[strept]avidinbiotin method as we described previously (Nagasue et al, 2000). An affinity-purified biotinylated secondary antibody in conjunction with streptavidin-peroxidase (for TFF2) and streptavidin-alkaline phosphatase (for $\alpha$-SMA) was used. In between the staining of the two proteins, slides were microwaved $(5$ minutes $\times 2)$ to prevent the cross-reactivity of the antibodies.

\section{Northern Blot Analysis}

Frozen tissues suitable for RNA extraction were available in 31 patients and were used for Northern blot analysis. Total RNA was extracted from tumor and corresponding normal mucosa samples using the TRI reagent (Molecular Research Center, Inc., Cincinnati, Ohio) following the manufacturer's instructions. Samples of $20 \mu \mathrm{g}$ of total RNA were resolved by electrophoresis on $1 \%$ formaldehyde-agarose gels and transferred to nylon membranes. The integrity of the RNA was checked by ethidium bromide staining of the gel before transfer to the nylon membrane. The blots were baked at $80^{\circ} \mathrm{C}$ for 2 hours and were prehybridized with rapid-hyb buffer (Amersham Pharmacia Biotech UK Limited, Little Chalfont Buckinghamshire, England) at $65^{\circ} \mathrm{C}$ for 15 minutes. A full-length cDNA of TFF2 was amplified by nested PCR and cloned into a TA cloning vector (pGEM-T Easy Vector Systems; Promega, Madison, Wisconsin). The sequence of the insert was confirmed with a DNA sequencer (Applied Biosystems, Newark, Connecticut; Sawade Technology Ltd., Tokyo, Japan). Hybridization was done with the same prehybridization solution in the presence of denatured [ $\left.{ }^{32} \mathrm{P}\right]$ radiolabeled (rediprime II, Amersham Pharmacia Biotech UK Limited) probe for 18 hours at $65^{\circ} \mathrm{C}$. After three stringency washes, the blots were exposed to imaging plates (Fuji Photo Film Company, Ltd., Tokyo, Japan) overnight, and the signals were processed by the BAS 2000 II (Fujix Bas-station, version 1.31, Fuji Photo Film Company) for quantification of mRNA. Signals were corrected for $28 \mathrm{~S}$ signals generated by ethidium bromide staining of the gel before transfer to the nylon membrane.

\section{In Situ Hybridization}

Sections from selected cases having positive $(n=10)$ and negative $(n=10)$ TFF2 expression by immunohistochemical analysis were used for in situ hybridization. Parallel sections were used from each patient for sense and antisense probe. A full-length cDNA of TFF2 was used for the probe construction. A 
digoxigenin-labeled sense and antisense RNA probe was transcribed by T7 and SP6 RNA polymerase, respectively, with a DIG RNA labeling kit according to the manufacturer's instructions (Boehringer Mannheim, Mannheim, Germany). Hybridization and the immunohistochemical steps were done as we described previously (Nagasue et al, 2000).

\section{Statistical Analysis}

The correlation between TFF2 expression and various clinicopathologic parameters was determined by the Fisher's exact test or Mann-Whitney $U$ test as appropriate. The correlation between the immunohistochemical data and the Northern blot data was examined by the Spearman's rank test. The survival curves were plotted using the Kaplan-Meier method, and the statistical significance between groups was determined by the log rank test. The end points for analysis were the disease-free survival and the overall survival starting from the day of operation. Independent variables predicting survival were evaluated by the multiple stepwise regression analysis using the Cox model. The Statview 4.5J (Abacus Concepts, Berkeley, California) software was used for data analysis.

\section{References}

Alison MR, Chinery R, Poulsom R, Ashwood P, Longcroft JM, and Wright NA (1997). Experimental ulceration leads to sequential expression of spasmolytic polypeptide, intestinal trefoil factor, epidermal growth factor and transforming growth factor alpha mRNAs in rat stomach. J Pathol 175: 405-414.

Dignass A, Lynch-Devaney K, Kindon H, Thim L, and Podolsky DK (1994). Trefoil peptides promote epithelial migration through a transforming growth factor-independent pathway. $\mathrm{J}$ Clin Invest 94:376-383.

Efstathiou JA, Liu D, Wheeler JMD, Kim HC, Beck NE, llyas M, Karayiannakis AJ, Mortensen NJ, Kmiot W, Playford RJ, Pignatelli M, and Bodmer WF (1999). Mutated epithelial cadherin is associated with increased tumorigenicity and loss of adhesion and of responsiveness to the motogenic trefoil factor 2 in colon carcinoma cells. Proc Natl Acad Sci USA 96:2316-2321.

Elia G, Chinery R, Hanby AM, Poulsom R, and Wright NA (1994). The production and characterization of a new monoclonal antibody to the trefoil peptide human spasmolytic polypeptide. Histochem J 26:644-647.

Emami S, Floch NL, Bruyneel E, Thim L, May F, Westley B, Rio MC, Mareel M, and Gespach C (2001). Induction of scattering and cellular invasion by trefoil peptides in src- and RhoA-transformed kidney and colonic epithelial cells. FASEB J 15:351-361.

Farrel JJ, Taupin D, Koh TJ, Chen D, Zhao CM, Podolsky DK, and Wang TC (2002). TFF2/SP-deficient mice show decreased gastric proliferation, increased acid secretion, and increased susceptibility to NSAID injury. J Clin Invest 109: 193-204.

Graness A, Chwieralski CE, Reinhold D, Thim L, and Hoffmann W (2002). Protein kinase $C$ and ERK activation are required for TFF-peptide-stimulated bronchial epithelial cell migration and tumor necrosis factor-alpha-induced interleukin-6 (IL-6) and IL-8 secretion. J Biol Chem 24: 18440-18446.

Halldorsdottir AM, Sigurdardottrir M, Jonasson JG, Oddsdottir M, Magnusson J, Lee JR, and Goldenring JR (2003). Spasmolytic polypeptide-expressing metaplasia (SPEM) associated with gastric cancer in Iceland. Dig Dis Sci 48:431-441.

Hanby AM, Chinery R, Poulsom R, Playford RJ, and Pignatelli $M$ (1996). Downregulation of E-cadherin in the reparative epithelium of the hum an gastrointestinal tract. Am J Pathol 148:723-729.

Hoosein NM, Thim L, Jorgensen KH, and Brattain MG (1989). Growth stimulatory effect of pancreatic spasmolytic polypeptide on culture colon and breast tumor cells. FEBS Lett 247:303-306.

Jørgensen KD, Diamant B, Jørgensen KH, and Thim L (1982). Pancreatic spasmolytic polypeptide (PSP): III. Pharmacology of a new porcine pancreatic polypeptide with spasmolytic and gastric acid secretion inhibitory effects. Regul Pept 3:231-242.

Kirikoshi $\mathrm{H}$ and Katoh M (2002). Expression of TFF1, TFF2 and TFF3 in gastric cancer. Int J Oncol 21:655-659.

Lefebvre O, Chenard MP, Masson R, Linares J, Dierich A, LeMeur M, Wendling C, Tomasetto C, Chambon P, and Rio MC (1996). Gastric mucosa abnormalities and tumorigenesis in mice lacking the pS2 trefoil protein. Science 274:259-262.

Leung WK, Yu J, Chan FK, To KF, Chan MW, Ebert MP, Ng EK, Chung SC, Malfertheiner P, and Sung JJ (2002). Expression of trefoil peptides (TFF1, TFF2, and TFF3) in gastric carcinomas, intestinal metaplasia, and non-neoplastic gastric tissues. J Pathol 197:582-588.

Liu D, el-Hariry I, Karayiannakis AJ, Wilding J, Chinery R, Kmiot W, McCrea PD, Gullick WJ, and Pignatelli M (1997). Phosphorylation of beta-catenin and epidermal growth factor receptor by intestinal trefoil factor. Lab Invest 77:557-563.

Machado JC, Nogueira AMMF, Carneiro F, Reis CA, and Sobrinho-Simoes M (2002). Gastric carcinoma exhibit distinct types of cell differentiation: An immunohistochemical study of trefoil peptides (TFF1 and TFF2) and mucins (MUC1, MUC2, MUC5AC, and MUC6). J Pathol 190:437-443.

Nagasue N, Dhar DK, Yamanoi A, Emi Y, Udagawa J, Yamamoto A, Tachibana M, Kubota H, Kohno H, and Harada $T$ (2000). Production and release of endothelin-1 from the gut and spleen in portal hypertension due to cirrhosis. Hepatology 31:1107-1114.

Otto WR, Rao J, Cox HM, Kotzain E, Lee C, Goodlad RA, Lane A, Gorman M, Freemont PA, Hansen HF, Pappin D, and Wright NA (1996). Effects of pancreatic spasmolytic polypeptide (PSP) on epithelial cell function. Eur J Biochem 235:64-72.

Park WS, Oh RR, Park JY, Lee JH, Shin MS, Kim HS, Lee HK, Kim YS, Kim SY, Lee SH, Yoo NJ, and Lee JY (2000). Somatic mutations of the trefoil factor family 1 gene in gastric cancer. Gastroenterology 119:691-698.

Playford RJ, Marchbank T, Chinery R, Evison R, Pignatelli M, Boulton RA, Thim L, and Hanby AM (1995). Human spasmolytic polypeptide is a cytoprotective agent that stimulates cell migration. Gastroenterology 108:108-116.

Schmidt PH, Lee JR, Joshi V, Playford RJ, Poulsom R, Wright NA, and Goldenring JR (1999). Identification of a metaplastic cell lineage associated with human gastric adenocarcinoma. Lab Invest 79:639-646. 
Seib T, Blin N, Hilgert K, Seifert M, Theisinger B, Engel M, Dooley S, Zang KD, and Welter C (1997). The three human trefoil genes TFF1, TFF2, and TFF3 are located within a region of $55 \mathrm{~kb}$ on chromosome 21q22.3. Genomics 40:200-202.

Sobin LH and Wittekind $\mathrm{CH}$, editors (1997). International Union Against Cancer (UICC). TNM classification of malignent tumors, 5th ed. New York: Wiley.

Taupin D, Wu DC, Jeon WK, Devaney K, Wang TC, and Podolsky DK (1999). The trefoil gene family are coordinately expressed immediate-early genes: EGF receptor and MAP kinase-dependent interregulation. J Clin Invest 103:R31R38.

Theisinger B, Welter C, Seitz G, Rio MC, Lathe R, Chambon $P$, and Blin N (1991). Expression of the breast cancer associated gene pS2 and pancreatic spasmolytic polypeptide gene (hSP) in diffuse type of stomach carcinoma. Eur J Cancer 27:770-773.
Wang TC, Goldenring JR, Dangler CA, Ito S, Muller A, Jeon WK, Koh TJ, and Fox JG (1998). Mice lacking secretory phospholipase A2 show altered apoptosis and differentiation with $\mathrm{H}$ felis infection. Gastroenterology 114:675-689.

Wang TC, Dangler CA, Chen D, Goldenring JR, Koh T, Raychowdhury R, Coffey RJ, Ito S, Varro A, Dockray GJ, and Fox JG (2000). Synergistic interaction between hypergastrinemia and Helicobacter infection in a mouse model of gastric cancer. Gastroenterology 118:36-47.

Wright NA, Hoffmann W, Otto WR, Rio MC, and Thim L (1997). Rolling in the clover: Trefoil factor family (TFF)-domain peptides, cell migration and cancer. FEBS Lett 408:121-123.

Yamachika T, Werther JL, Bodain C, Babyyatsky M, Tatematsu M, Yamamura Y, Chen A, and Itzkowitz S (2002). Intestinal trefoil factor: A marker of poor prognosis in gastric carcinoma. Clin Cancer Res 8:1092-1099. 